\title{
$<$ CATEG $>$ COMMENTARY
}

\section{MARTIN AND SEEMAN}

\section{DOES ABALOPARATIDE SPARE RESORPTION?}

\section{Abaloparatide Is an Anabolic, but Does It Spare Resorption?}

T Jack Martin ${ }^{1,2}$ and Ego Seeman ${ }^{3,4}$

${ }^{1}$ St Vincent's Institute of Medical Research, Melbourne, Australia

${ }^{2}$ Department of Medicine, St Vincent's Health, University of Melbourne, Melbourne, Australia

${ }^{3}$ Department of Medicine, Austin Health, University of Melbourne, Melbourne, Australia

${ }^{4}$ Department of Health and Ageing, Australian Catholic University, Melbourne, Australia

Address correspondence to:

This is a Commentary on Miller et al. (JAMA. 2016;316(7):722-33. DOI:

10.1001/jama.2016.11136) and Cosman et al. (J Bone Miner Res. 2017;32(XX):XXX-

XXX.<zaq;3> DOI: 10.1002/jbmr.2991)

Journal of Bone and Mineral Research, Vol. 32, No. 00, Month 2017, pp 0000-0000

DOI: $10.1002 / j b m r .3042$

(C) 2017 American Society for Bone and Mineral Research</FNTX>

Longevity is increasing the burden of fractures. Although antiresorptive therapy slows remodeling, it does not prevent or repair microstructural deterioration. The challenge is to reconstruct the skeleton. The more advanced the patient's age, the more likely that microstructural deterioration is severe and so the more rational the choice of an anabolic agent as first-line therapy. PTH(1-34) (teriparatide) reduces vertebral and nonvertebral fracture risk, in part, by increasing bone matrix volume. Abaloparatide has also recently been reported to reduce morphometric vertebral fracture risk $^{(1,2)}$ and is thus a most welcome addition to the therapeutics of bone fragility. Abaloparatide is claimed to produce its anabolic effect with a much lesser effect on bone resorption than occurs with teriparatide. In this Commentary, we query the latter proposition, which we suggest contains two misconceptions: first, that with teriparatide treatment an anabolic window exists during which bone formation predominates but then is offset by bone resorption, which follows later, and second that less bone resorption occurs with abaloparatide, leaving the "window" open longer and allowing more bone formation.

\section{Anabolic Therapy With Parathyroid Hormone (PTH) and Abaloparatide}


To understand the fallacies in these two notions, the mythical anabolic window and the purported lesser resorptive effect of abaloparatide, it is necessary first to discuss the mechanisms of action of PTH. The idea that intermittent injection of PTH might promote bone formation was proposed more than 80 years ago by Albright ${ }^{(3)}$ and shown in rats by Selye. ${ }^{(4)}$ Progress was slow, with no attempt to study PTH treatment clinically until the 1970s. The first clinical trial was published in $2001^{(5)}$ and was stopped after 18 months because of a toxicity study that showed dose-dependent development of osteosarcoma in rats treated throughout their lives. ${ }^{(6)}$ With the total number of subjects in the clinical trial reduced to 1600 and the duration of treatment being only 18 months, no potential antihip fracture efficacy could be demonstrated, but vertebral and nonvertebral fracture risk was reduced and bone mineral density (BMD) increased.

The efficacy of teriparatide as an anabolic therapy depends on intermittent injections, each achieving a sharp peak of PTH in the blood. ${ }^{(7)}$ When teriparatide therapy is begun, circulating bone formation markers increase rapidly, with a delayed appearance of resorption markers, giving rise to the concept of the "anabolic window." ${ }^{(8,9)}$ This was based on the idea that the initial period of increased anabolic markers reflects an early period of bone formation relatively "unopposed" by resorption but then superseded by subsequent increases in bone resorption. This theory was not based on histomorphometric evidence of changing volumes of bone formation and resorption but rather inferred from finding an early increase in bone "formation" markers, followed by a later increase in bone "resorption" markers.

Abaloparatide is a 34-amino acid peptide acting upon the PTH/PTHrP receptor (PTHR1) that has been reported in a new study to be comparable with teriparatide in reducing morphometric vertebral fractures in postmenopausal women. ${ }^{(1,2)}$ It is claimed to act with a lesser effect on resorption markers than that with teriparatide. ${ }^{(1,2,10)}$ The first 21 residues are identical with parathyroid hormone-related protein (PTHrP), and the remainder contain a number of substitutions that were planned to improve stability. ${ }^{(11,12)}$ These structural features of abaloparatide have led to it being called a PTHrP analog that is a selective activator of the PTHR1. ${ }^{(10-12)}$ Abaloparatide and teriparatide have now been reported to have comparable antivertebral fracture efficacy with a possible earlier and greater effect of abaloparatide in reducing major osteoporotic fractures, an effect suggested to be the result a greater net bone formation due to a lesser effect on bone resorption than teriparatide. ${ }^{(1,2,10)}$ 
The anabolic effect of teriparatide is predominantly remodeling based in human subjects. ${ }^{(13,14)}$ The delay in resorption marker increase after starting PTH treatment can be explained by the principles of basic multicellular unit (BMU)-based remodeling. ${ }^{(15-18)}$ BMUs remodeling bone are in their resorptive, reversal, or formative phases. At any time, there are more BMUs in their formation phase at various locations than there are BMUs in their resorption phase at other locations (because the duration of the formation phase is longer than the resorption phase). ${ }^{(19)}$

Teriparatide acting on BMUs in their reversal or formation phase promotes differentiation of osteoblast lineage cells into mature osteoid-producing forms, while inhibiting osteoblast apoptosis. ${ }^{(20)}$ The net effect is the deposition of a volume of bone and an increase in P1NP. If the volume of bone deposited exceeds the volume of bone excavated (weeks to months earlier), trabecular and cortical thickness increase at that location. ${ }^{(20-22)}$ The early rise in P1NP may also be the result of a direct modeling-based effect of teriparatide as well ${ }^{(14,23)}$ (Fig. 1). \{FIG1 \} Teriparatide also acts on BMUs in their resorptive phase and generates new BMUs that must first enter their resorptive phase; this results from teriparatide promotion of osteocyte and osteoblast precursor production of receptor activator of NF- $\mathrm{BB}$ ligand (RANKL), osteoclastogenesis, and bone resorption. When enough matrix has been resorbed, eventually resorption markers reach a measurable level in the circulation. This provides an explanation for the delay in the increase in resorption markers relative to formation markers after the start of teriparatide treatment, without the need to invoke a "window" of exclusively anabolic action.

Increased resorption markers are unlikely to signal the end of bone formation because these newly generated BMUs in their resorption phase proceed into their reversal phase during which coupling facilitates bone formation, a process taking several months. An end of the anabolic effect of teriparatide is what the term "window" implies. Dempster and colleagues suggest the contrary with continued bone formation produced by teriparatide during 2 years, well after the increase in bone "resorption" markers. ${ }^{(23)}$

\section{Why Consider PTHrP as an Anabolic Ligand?}

The development of abaloparatide draws much on what is known of the actions of PTHrP(1-36), the aminoterminal portion of PTHrP. Although PTHrP was discovered as a circulating factor that causes the humoral hypercalcemia of malignancy, it does not circulate as a hormone postnatally but is produced by many tissues in the body, where it acts as an autocrine and paracrine regulator. ${ }^{(24,25)}$ This includes bone, where it is produced by cells within the osteoblast lineage. ${ }^{(26-29)}$ PTHrP 
homology with PTH is restricted to the amino-terminal region, equipping them to act with equivalent potencies on the common receptor, PTHR $1 .{ }^{(30,31)}$ PTHrP has other actions mediated by unique regions within the molecule beyond the amino-terminal region that is homologous with PTH. ${ }^{(32)}$

Studies in genetically manipulated mice have been crucial in informing us of the roles of PTHrP in bone. Mice in which the PTHrP gene was ablated were born with severe skeletal abnormalities, indicating a requirement for PTHrP in endochondral bone formation. ${ }^{(33)}$ Haploinsufficient $\mathrm{PTHrP}(+/-)$ mice that were phenotypically normal at birth were found to be osteopenic by 3 months of age, ${ }^{(34)}$ a phenotype recapitulated in mice with an osteoblast-specific knockout of PTHrP. ${ }^{(35)}$ These genetic experiments revealed that PTHrP is a physiologic regulator of bone formation that stimulates bone formation by promoting the differentiation of committed osteoblast precursors and by inhibiting apoptosis of mature osteoblasts and osteocytes. ${ }^{(20,36)}$ These actions of PTHrP are known pharmacological effects of teriparatide.

With the recognition that PTHrP is the paracrine ligand for the PTHR1 in bone, several truncated forms were investigated as anabolic agents, including $\mathrm{PTHrP}(1-34)$, $\mathrm{PTHrP}(1-36)$, and $\mathrm{PTHrP}(1-$ 74). From the earliest of these studies, ${ }^{(37)}$ it was evident that PTHrP is significantly less potent as an anabolic agent in vivo than PTH, although the two are equally active in promoting cAMP production in target cells. This was anticipated because PTHrP has many target sequences within its structure that are particularly susceptible to proteolysis. ${ }^{(32,38,39)}$ One of the most susceptible of these sequences is $-\mathrm{R}_{19} \mathrm{R}_{20} \mathrm{R}_{21^{-}},{ }^{(39)}$ which is located within both $\mathrm{PTHrP}(1-36)$ and abaloparatide. Thus, it is plausible that inactivation after subcutaneous injection would reduce the amount of agonist presented to receptor targets. In other words, abaloparatide is likely to function as a weaker agonist of the PTHR1 receptor.

\section{Are PTHrP(1-36) and Abaloparatide Anabolic and Resorption Sparing?}

The anabolic action of PTHrP(1-36) in human subjects has been investigated extensively by Stewart and colleagues, who suggested it was relatively free of a resorptive effect as determined by measuring bone formation and resorption markers. ${ }^{(7,40-43)}$ In these studies of PTHrP(1-36), the daily injection dose in human subjects required to increase levels of formation markers was many-fold higher than the dose of teriparatide needed to achieve these marker levels. ${ }^{(43,44)}$ As discussed below and elsewhere, resorption and formation "markers" are not accurate surrogates of volumes of bone matrix resorbed or formed. ${ }^{(18,45)}$ Whether PTHrP(1-36) and teriparatide produce 
a similar anabolic effect but $\mathrm{PTHrP}(1-36)$ produces less bone resorption than teriparatide was not evaluated; no histomorphometry was performed. However, a recent large study of iliac crest biopsies from the Miller and colleagues study ${ }^{(1)}$ reported no differences in static or dynamic indices of bone formation or resorption between the two drugs. ${ }^{(46)}$ The possibility that PTHrP(1-36) is anabolic while sparing of resorption would be intriguing but difficult to explain given resorption is an inescapable first phase of bone remodeling. One possible explanation for a resorption-sparing anabolic effect might be that the higher dose requirement is owing to a difference in pharmacokinetics, with PTHrP(1-36) being degraded more rapidly after injection and thus not so widely distributed to activate BMUs. However, if less agonist is available to the receptor, then this should lead to less anabolic and resorptive effects.

Data reported by Miller and colleagues ${ }^{(1)}$ and in the related article in this issue of $J B M R^{(2)}$ identify abaloparatide as an effective approach to fracture risk reduction. The study by Miller and colleagues $^{(1)}$ used $80 \mu \mathrm{g}$ per day of abaloparatide and $20 \mu \mathrm{g}$ of teriparatide. These differing doses were probably chosen as a consequence of the experience with $\operatorname{PTHrP}(1-36)$, where much higher doses were required to produce an anabolic effect. The dosage equivalence of these agents remains uncertain. The incidence of hypercalcemia was significantly higher with teriparatide (6.4\%) than with abaloparatide $(3.4 \%, p=0.006){ }^{(1)}$ In examining this further, the question of relative dosage will need to be resolved.

Miller and colleagues report that both resorption and formation markers increased less with abaloparatide than with teriparatide. However, the authors claim that abaloparatide has a seemingly more favorable net formation-resorption marker profile. Namely, the increase in resorption markers was less relative to the increase in formation markers than the relative changes in formationresorption markers observed in the teriparatide group. The authors infer that this may account for the $1 \%$ higher BMD at proximal femur sites. Leder and colleagues ${ }^{(10)}$ make similar inferences; the relative changes in the "resorption" and "formation" markers may account for the $2 \%$ difference in BMD, a study of 24 weeks' duration.

If serum biochemical markers are to be used as surrogates for volumes of bone matrix resorbed and formed, then the formation marker data for abaloparatide in Miller and colleagues ${ }^{(1)}$ predict less bone formation, despite the fracture risk reduction. An alternative interpretation of the data is that less of the undegraded abaloparatide peptide reaches the PTHR1 in appropriate cells in bone. Greater susceptibility to inactivation of injected material could be predicted, as discussed above, 
leading to abaloparatide functioning as a less potent agonist than teriparatide but nevertheless capable of achieving fracture reduction, as in the abaloparatide clinical trial.

\section{Receptor Interaction and Clearance of Abaloparatide}

Another explanation has been proposed for this suggested difference between abaloparatide and PTH, based on studies of interaction with the PTHR1 of teriparatide and PTHrP(1-36). In cells engineered to overexpress PTH1R, teriparatide and $\mathrm{PTHrP}(1-36)$ differed in their initial receptor interaction mechanisms; although they increased cAMP with comparable potencies, the action of $\mathrm{PTHrP}(1-36)$ was restricted to the cell surface, whereas teriparatide was more readily internalized and brought about a somewhat more prolonged increase in cyclic AMP in the target cells. ${ }^{(47)}$ The authors suggested that such a differential response might explain why daily injection of teriparatide exhibits a resorption response, whereas $\mathrm{PTHrP}(1-36)$ does so to a lesser extent. The same argument has been applied to abaloparatide, ${ }^{(1,2)}$ which behaves like PTHrP(1-36) in the study of initial interaction with receptor. ${ }^{(12)}$ These findings of differing initial interactions of the peptides with receptor provide interesting cell biology, but extrapolating them to explain the purported in vivo effects is questionable given the lack of evidence of differences in cellular effects beyond cAMP generation.

\section{Abaloparatide Is an Anabolic Agent, but Is It Relatively Resorption-Free?}

Also published in this issue of $J B M R$ is the first preclinical study of the anabolic effect of abaloparatide. ${ }^{(48)}$ This was carried out in rats, a mammalian species that models its skeleton throughout life with minimal remodeling of metaphyseal trabecular bone. Claims of a lesser resorptive effect of abaloparatide are problematic because the authors used the aged osteopenic rat that undergoes an anabolic response to PTH without any evidence of a remodeling effect. In this animal model, it has been shown repeatedly that anabolic therapy through the PTH/PTHrP receptor is achieved without any evidence of resorption activation. This has been assessed using both biochemical markers and histomorphometry and implies that the induced formation is taking place predominantly on quiescent surfaces. ${ }^{(49-57)}$

The same outcome is reported by Varela and colleagues, ${ }^{(48)}$ where aged osteopenic rats were treated with three doses of abaloparatide $(1,5$, and $25 \mu \mathrm{g} / \mathrm{kg})$, resulting in substantial dose-responsive increases in BMD and bone formation, assessed by histomorphometry, without any significant increase in resorption parameters. It is interesting that such low doses of abaloparatide were used in 
this study, given the choice in the clinical trial of a dose 4 times higher than that of teriparatide, and the known very much higher dose of PTHrP(1-36) required in mice and in human subjects. These doses of abaloparatide are in the same range of dose-dependent effects observed in studies of PTH(1-34) in aged osteopenic rats, where similar dose-responsive anabolic effects with PTH(1-34) were achieved at $0.1,0.3,3$, and $30 \mu \mathrm{g} / \mathrm{kg}^{(49)}$ and with 5 and $15 \mu \mathrm{g} / \mathrm{kg}$. ${ }^{(50)}$ In these latter two studies and many other studies in aged ovariectomized (OVX) rats, some using low PTH doses, others using relatively high doses $(100 \mu \mathrm{g} / \mathrm{kg}){ }^{(51-57)}$ the anabolic effect was achieved without any increase in osteoclast surface or resorption markers.

The lack of remodeling is also apparent in vehicle-treated OVX controls in the study by Varela and colleagues. ${ }^{(48)}$ There was no decrease in total or regional BMD and no increase in activation frequency or osteoclast-covered surfaces after OVX. Trabecular numbers decreased, but there was no evidence of reduced cortical width or area or increased endocortical eroded surface.

Abaloparatide was indeed anabolic. Bone formation increased upon trabecular, endocortical, and periosteal surfaces and bone formation markers increased. This is a modeling-based anabolic effect-it proceeds without increasing eroded surfaces, osteoclast numbers, or bone resorption markers. Although this is the outcome of abaloparatide treatment in the present study, ${ }^{(48)}$ the findings cannot be used to argue for a different mechanism of action of abaloparatide. Nothing can be claimed of the relative effects on resorption of abaloparatide and teriparatide in the rat without a direct comparison in the same experiment. Moroever, an experimental model other than the aged, osteopenic rat is required to address the hypothesis that abaloparatide will increase bone formation without increasing bone resorption in human subjects where the response to teriparatide treatment is predominantly remodeling based.

Varela and colleagues write in detail of an "anabolic window" and even calculate it from differences between "resorption" and "formation" markers in rats "to evaluate treatment-related changes in the relative balance of bone formation and resorption," ${ }^{(48)}$ despite the response of the rats to PTH being a modeling one in which resorption and formation are not coupled; they do not become "unbalanced"- - they proceed at different locations.

\section{There Is No Anabolic Window to Open or Close}

The difference in the volumes of matrix resorbed and then formed by each BMU determine whether there is net loss or gain in matrix volume. There are formidable challenges in determining whether less bone is resorbed using abaloparatide than teriparatide when remodeling markers are used as 
surrogates of these cellular events. ${ }^{(18,45)}$ The biochemical measurements correlate weakly with radiokinetic measurements of remodeling or the surface extent of bone remodeling determined using histomorphometry. ${ }^{(58,59)}$ No correlations with measures of the volumes of bone resorbed or deposited upon a skeletal surface have been reported.

The early formation marker increase in response to PTHR1 activation is a relatively rapid cell response. After sufficient matrix production has taken place, resorption markers such as CTX eventually reach levels measurable in the circulation, providing a ready explanation for the delayed increase in resorption markers. Moreover, differences in concentration of the markers in a blood sample are determined more by differences in remodeling rate and so the surface extent of remodeling rather than differences in BMU balance. Neither of the surface extents of resorption and formation inform as to whether there is net removal of, or deposition upon, the periosteal, intracortical, endocortical, or trabecular surfaces.

In addition, the use of differences in "resorption" and "formation" markers to estimate the change in femoral neck BMD in a 24 -week clinical study ${ }^{(10)}$ or total body bone mineral content (BMC) in the rat study ${ }^{(48)}$ takes no account of the many mechanisms that contribute to a rise in BMD. Indeed, BMD often decreases during teriparatide therapy as bone matrix volume increases, in part, because of an increase in porosity and the replacement of older, more mineralized bone with a larger volume of younger, initially less mineralized bone matrix. ${ }^{(60)}$ The rise in BMD is the net result of an increase in bone matrix volume, any modeling-based bone formation, and the mineralization of that matrix that is independent of the remodeling or modeling activity captured by the remodeling marker.

\section{Do Abaloparatide and Teriparatide Differ in Their Antifracture Efficacy?}

Abaloparatide is claimed to produce "early increases in BMD and earlier fracture protection" as a consequence of "earlier and greater net bone formation than resorption" than teriparatide. The claim of earlier fracture protection is partly the result of women randomized to teriparatide or placebo having fractures within weeks of initiation of the trial, whereas those allocated to abaloparatide did not. Were these early fractures the result of lack of efficacy of teriparatide and the absence of fractures attributable to efficacy of abaloparatide? The more likely explanation is that these early fractures are fortuitous because mechanisms believed to reduce fracture risk are unlikely to be operative. Nevertheless, these events were included in the analysis and contributed to finding significantly fewer major fragility fractures in the abaloparatide group $(n=4)$ than the teriparatide 
group $(n=13)$. Between 6 and 12 months, there were 5 fractures in the abaloparatide and 4 fractures in the teriparatide groups. Between 12 and 18 months, there were 1 and 6 events in the respective groups. Identifying an advantage of one drug over another is challenging, particularly when events are uncommon and when uneven loss of the inception cohort may compromise the fidelity of randomization.

\section{Conclusion}

Even if there were convincing evidence that abaloparatide stimulates bone formation with a lesser accompanying increase in resorption markers than with teriparatide, the hypothesis has a significant drawback. There is compelling evidence ${ }^{(13,14,23,61)}$ that the anabolic effect of PTH through PTHR1 in human subjects is mainly (70\%) through increasing the number and activity of BMUs, as well as recent evidence ${ }^{(23)}$ that there is also some modeling effect on the periosteal surface. Therefore, if abaloparatide is acting through the PTHR1 and the PTH "anabolic" pathway, remodeling is required, and of course resorption is an inescapable accompaniment of that process, because the PTH anabolic mechanism requires the recruitment and activation of new BMUs. Logically, if there is less remodeling, there will be a lesser anabolic response.

We are entering the era of anabolic therapy. Abaloparatide can be a contribution to the therapeutics of bone fragility, as can romosozumab. ${ }^{(62)}$ It is an essential step given population demographics and the increasingly recognized limitations of antiresorptives. We are also entering an era where we can quantify bone's material composition and microstructure. This is also an essential step, away from the "one treatment fits all" approach of the last century and much of this one, and away from the vagaries of BMD that obscure concomitant and opposite changes in matrix mineral density and matrix volume, creating confusion. The more rational approach is toward defining mechanistic and morphological targets responsible for bone fragility.

The mechanism of action of abaloparatide is of interest, whether or not it is identical to that of teriparatide, and even if it is simply a less potent activator of the PTH anabolic pathway. Any proposal that abaloparatide has a greater modeling effect than teriparatide would need to take into account the fact that the two are acting through the same receptor. Resolution of this will require much more work, which should include challenging experiments such as using quantitative histomorphometry of bone in dose-response studies from remodeling species and from treated human subjects. ${ }^{(13,14,23)}$ This will continue as part of the unfolding story of how an anabolic effect on the skeleton is achieved through the PTHR1 receptor. 


\section{Disclosures}

The authors state that they have no conflicts of interest. <zaq;4>

\section{Acknowledgments}

Research from the authors' laboratories was supported by grants from the NHMRC, Australia, and to St Vincent's Institute through the Victorian Government OIS Program. The authors acknowledge helpful comments from Natalie Sims.

\section{References}

1. Miller PD, Hattersley G, Riis BJ, et al. Effect of abaloparatide vs placebo on new vertebral fractures in postmenopausal women with osteoporosis: a randomized clinical trial. JAMA. 2016;316(7):722-33.

2. Cosman F, Hattersley G, Hu MY, Williams GC, Fitzpatrick LA, Black DM. Effects of abaloparatide-SC on fractures and bone mineral density in subgroups of postmenopausal women with osteoporosis and varying baseline risk factors. J Bone Miner Res. 2016;32(XX):XXXXXX. <zaq;3>

3. Albright F, Bauer W, Ropes M, Aub J. Studies of calcium and phosphorus metabolism: IV. The effect of the parathyroid hormone. J Clin Invest. 1929;7(1):139-81.

4. Selye H. On the stimulation of new bone formation with parathyroid extract and irradiated ergosterol. Endocrinology. 1932;16:547-58.

5. Neer RM, Arnaud CD, Zanchetta JR, et al. Effect of parathyroid hormone (1-34) on fractures and bone mineral density in postmenopausal women with osteoporosis. N Engl J Med. 2001;344(19):1434-41.

6. Vahle JL, Sato M, Long GG, et al. Skeletal changes in rats given daily subcutaneous injections of recombinant human parathyroid hormone (1-34) for 2 years and relevance to human safety. Toxicol Pathol. 2002;30(3):312-21.

7. Frolik CA, Black EC, Cain RL, et al. Anabolic and catabolic bone effects of human parathyroid hormone (1-34) are predicted by duration of hormone exposure. Bone. 2003;33(3):372-9.

8. Rubin MR, Bilezikian JP. Parathyroid hormone as an anabolic skeletal therapy. Drugs. 2005;65(17):2481-98.

9. Bilezikian JP. Combination anabolic and antiresorptive therapy for osteoporosis: opening the anabolic window. Curr Osteoporos Rep. 2008;6(1):24-30. 
10. Leder BZ, O'Dea LS, Zanchetta JR, et al. Effects of abaloparatide, a human parathyroid hormone-related peptide analog, on bone mineral density in postmenopausal women with osteoporosis. J Clin Endocrinol Metab. 2015;100(2):697-706.

11. Dong J, Shen Y, Culler M, et al. Highly potent analogs of human parathyroid hormone and human parathyroid hormone-related protein. In: Houghten RL, Lebl M, editors. Peptides: the wave of the future. American Peptide Society; 2001. p. 668-9.

12. Hattersley G, Dean T, Corbin BA, Bahar H, Gardella TJ. Binding selectivity of abaloparatide for PTH-type-1-receptor conformations and effects on downstream signaling. Endocrinology. 2016;157(1):141-9.

13. Lindsay R, Cosman F, Zhou H, et al. A novel tetracycline labeling schedule for longitudinal evaluation of the short-term effects of anabolic therapy with a single iliac crest bone biopsy: early actions of teriparatide. J Bone Miner Res. 2006;21(3):366-73.

14. Ma YL, Zeng Q, Donley DW, et al. Teriparatide increases bone formation in modeling and remodeling osteons and enhances IGF-II immunoreactivity in postmenopausal women with osteoporosis. J Bone Miner Res. 2006;21(6):855-64.

15. Frost HM. Dynamics of bone remodeling. In: Frost HM, editor. Bone biodynamics. Boston: Little, Brown; 1964. p. 315-33.

16. Parfitt AM. Skeletal heterogeneity and the purposes of bone remodelling: implications for the understanding of osteoporosis. In: Marcus R, Feldman D, Kelsey J, editors. Osteoporosis. San Diego, CA: Academic Press; 1996. p. 315-39.

17. Baron R, Tross R, Vignery A. Evidence of sequential remodeling in rat trabecular bone: morphology, dynamic histomorphometry, and changes during skeletal maturation. Anat Rec. 1984;208(1):137-45.

18. Seeman E, Martin TJ. Co-administration of antiresorptive and anabolic agents: a missed opportunity. J Bone Miner Res. 2015;30(5):753-64.

19. Parfitt A. Bone histomorphometry: techniques and interpretations. In: Recker RR, editor. Histomorphometry. Baton Rouge, LA: CRC Press; 1983. p. 142-221.

20. Jilka RL. Molecular and cellular mechanisms of the anabolic effect of intermittent PTH. Bone. 2007;40(6):1434-46.

21. Compston JE. Skeletal actions of intermittent parathyroid hormone: effects on bone remodelling and structure. Bone. 2007;40(6):1447-52. 
22. Dempster DW, Cosman F, Parisien M, Shen V, Lindsay R. Anabolic actions of parathyroid hormone on bone. Endocr Rev. 1993;14(6):690-709.

23. Dempster D, Zhou H, Recker RR, et al. Longitudinal changes in modelling- and remodelling-based bone formation with an anabolic vs an antiresorptive agent in the AVA Osteoporosis Study. J Bone Miner Res. 2016;31 (Suppl 1).

24. Martin TJ, Moseley JM, Williams ED. Parathyroid hormone-related protein: hormone and cytokine. J Endocrinol. 1997;154 Suppl:S23-37.

25. Philbrick WM, Wysolmerski JJ, Galbraith S, et al. Defining the roles of parathyroid hormone-related protein in normal physiology. Physiol Rev. 1996;76(1):127-73.

26. Moseley JM, Hayman JA, Danks JA, et al. Immunohistochemical detection of parathyroid hormone-related protein in human fetal epithelia. J Clin Endocrinol Metab. 1991;73(3):478-84.

27. Suda N, Gillespie MT, Traianedes K, et al. Expression of parathyroid hormone-related protein in cells of osteoblast lineage. J Cell Physiol. 1996;166(1):94-104.

28. Kartsogiannis V, Moseley J, McKelvie B, et al. Temporal expression of PTHrP during endochondral bone formation in mouse and intramembranous bone formation in an in vivo rabbit model. Bone. 1997;21(5):385-92.

29. Lee K, Deeds JD, Segre GV. Expression of parathyroid hormone-related peptide and its receptor messenger ribonucleic acids during fetal development of rats. Endocrinology. 1995;136(2):453-63.

30. Juppner H, Abou-Samra AB, Freeman M, et al. A G protein-linked receptor for parathyroid hormone and parathyroid hormone-related peptide. Science. 1991;254(5034):10246.

31. Hammonds RG Jr, McKay P, Winslow GA, et al. Purification and characterization of recombinant human parathyroid hormone-related protein. J Biol Chem. 1989;264(25):14806-11. 32. Martin TJ. Parathyroid hormone-related protein, its regulation of cartilage and bone development, and role in treating bone diseases. Physiol Rev. 2016;96(3):831-71.

33. Amizuka N, Warshawsky H, Henderson JE, Goltzman D, Karaplis AC. Parathyroid hormone-related peptide-depleted mice show abnormal epiphyseal cartilage development and altered endochondral bone formation. J Cell Biol. 1994;126(6):1611-23. 
34. Amizuka N, Karaplis AC, Henderson JE, et al. Haploinsufficiency of parathyroid hormone-related peptide (PTHrP) results in abnormal postnatal bone development. Dev Biol. 1996;175(1):166-76.

35. Miao D, He B, Jiang Y, et al. Osteoblast-derived PTHrP is a potent endogenous bone anabolic agent that modifies the therapeutic efficacy of administered PTH 1-34. J Clin Invest. 2005;115(9):2402-11.

36. Martin TJ. Osteoblast-derived PTHrP is a physiological regulator of bone formation. $\mathrm{J}$ Clin Invest. 2005;115(9):2322-4.

37. Hock JM, Fonseca J, Gunness-Hey M, Kemp BE, Martin TJ. Comparison of the anabolic effects of synthetic parathyroid hormone-related protein (PTHrP) 1-34 and PTH 1-34 on bone in rats. Endocrinology. 1989;125(4):2022-7.

38. Orloff JJ, Reddy D, de Papp AE, Yang KH, Soifer NE, Stewart AF. Parathyroid hormone-related protein as a prohormone: posttranslational processing and receptor interactions. Endocr Rev. 1994;15(1):40-60.

39. Diefenbach-Jagger H, Brenner C, Kemp BE, et al. Arg21 is the preferred kexin cleavage site in parathyroid-hormone-related protein. Eur J Biochem. 1995;229(1):91-8.

40. Horwitz MJ, Tedesco MB, Sereika SM, Hollis BW, Garcia-Ocana A, Stewart AF. Direct comparison of sustained infusion of human parathyroid hormone-related protein-(1-36) [hPTHrP-(1-36)] versus hPTH-(1-34) on serum calcium, plasma 1,25-dihydroxyvitamin D concentrations, and fractional calcium excretion in healthy human volunteers. J Clin Endocrinol Metab. 2003;88(4):1603-9.

41. Horwitz MJ, Tedesco MB, Sereika SM, et al. Safety and tolerability of subcutaneous PTHrP(1-36) in healthy human volunteers: a dose escalation study. Osteoporos Int. 2006;17(2):225-30.

42. Horwitz MJ, Tedesco MB, Garcia-Ocana A, et al. Parathyroid hormone-related protein for the treatment of postmenopausal osteoporosis: defining the maximal tolerable dose. J Clin Endocrinol Metab. 2010;95(3):1279-87.

43. Horwitz MJ, Augustine M, Kahn L, et al. A comparison of parathyroid hormone-related protein (1-36) and parathyroid hormone (1-34) on markers of bone turnover and bone density in postmenopausal women: the PrOP study. J Bone Miner Res. 2013;28(11):2266-76. 
44. Everhart-Caye M, Inzucchi SE, Guinness-Henry J, Mitnick MA, Stewart AF. Parathyroid hormone (PTH)-related protein(1-36) is equipotent to PTH(1-34) in humans. J Clin Endocrinol Metab. 1996;81(1):199-208.

45. Seeman E, Nguyen TV. Bone remodeling markers: so easy to measure, so difficult to interpret. Osteoporos Int. 2016;27(1):33-5.

46. Moreira C, Fitzpatrick L, Wang Y, Recker R. Effects of abaloparatide-SC (BA058) on bone histology and histomorphometry: the ACTIVE phase 3 trial. Bone. Epub 2016 Nov 5. DOI: 10.1016/j.bone.2016.11.004.

47. Ferrandon S, Feinstein TN, Castro M, et al. Sustained cyclic AMP production by parathyroid hormone receptor endocytosis. Nat Chem Biol. 2009;5(10):734-42.

48. Varela A, Chouinard L, Lesage E, Smith SY, Hattersley G. One year of abaloparatide, a selective activator of the PTH1 receptor, increased bone formation and bone mass in osteopenic ovariectomized rats without increasing bone resorption. J Bone Miner Res. 2016 Oct 17. DOI: 10.1002/jbmr.3003.

49. Sato M, Grese TA, Dodge JA, Bryant HU, Turner CH. Emerging therapies for the prevention or treatment of postmenopausal osteoporosis. J Med Chem. 1999;42(1):1-24. 50. Ma YL, Zeng QQ, Porras LL, et al. Teriparatide [rhPTH (1-34)], but not strontium ranelate, demonstrated bone anabolic efficacy in mature, osteopenic, ovariectomized rats. Endocrinology. 2011;152(5):1767-78.

51. Kimmel DB, Bozzato RP, Kronis KA, et al. The effect of recombinant human (1-84) or synthetic human (1-34) parathyroid hormone on the skeleton of adult osteopenic ovariectomized rats. Endocrinology. 1993;132(4):1577-84.

52. Liu CC, Kalu DN. Human parathyroid hormone-(1-34) prevents bone loss and augments bone formation in sexually mature ovariectomized rats. J Bone Miner Res. 1990;5(9):973-82. 53. Wronski TJ, Yen CF, Qi H, Dann LM. Parathyroid hormone is more effective than estrogen or bisphosphonates for restoration of lost bone mass in ovariectomized rats. Endocrinology. 1993;132(2):823-31.

54. Shen V, Dempster DW, Mellish RW, Birchman R, Horbert W, Lindsay R. Effects of combined and separate intermittent administration of low-dose human parathyroid hormone fragment (1-34) and 17 beta-estradiol on bone histomorphometry in ovariectomized rats with established osteopenia. Calcif Tissue Int. 1992;50(3):214-20. 
55. Ibbotson KJ, Orcutt CM, D'Souza SM, et al. Contrasting effects of parathyroid hormone and insulin-like growth factor I in an aged ovariectomized rat model of postmenopausal osteoporosis. J Bone Miner Res. 1992;7(4):425-32.

56. McManus JF, Davey RA, Maclean HE, et al. Intermittent Fugu parathyroid hormone 1 (1-34) is an anabolic bone agent in young male rats and osteopenic ovariectomized rats. Bone. 2008;42(6):1164-74.

57. Gooi JH, Chia LY, Walsh NC, et al. Decline in calcitonin receptor expression in osteocytes with age. J Endocrinol. 2014;221(2):181-91.

58. Eriksen EF, Charles P, Melsen F, Mosekilde L, Risteli L, Risteli J. Serum markers of type I collagen formation and degradation in metabolic bone disease: correlation with bone histomorphometry. J Bone Miner Res. 1993;8(2):127-32.

59. Weaver CM, Peacock M, Martin BR, et al. Quantification of biochemical markers of bone turnover by kinetic measures of bone formation and resorption in young healthy females. $\mathrm{J}$ Bone Miner Res. 1997;12(10):1714-20.

60. Vrahnas C, Pearson TA, Brunt AR, et al. Anabolic action of parathyroid hormone (PTH) does not compromise bone matrix mineral composition or maturation. Bone. 2016;93:146-54. 61. Dempster DW, Roschger P, Misof BM, et al. Differential effects of teriparatide and zoledronic acid on bone mineralization density distribution at 6 and 24 months in the SHOTZ Study. J Bone Miner Res. 2016;31(8):1527-35.

62. Cosman F, Crittenden D, Adachi J, et al. Romosozumab treatment in postmenopausal women with osteoporosis. N Engl J Med. 2016;375(16):1532-43.

Figure caption

Fig. 1. Sites of action of teriparatide and abaloparatide. These agents produce remodeling-based bone formation by acting on the reversal or formation phases of existing basic multicellular units (BMUs) and initiating new BMUs. They also act on quiescent bone surfaces producing a modeling-based bone formation. See text for details.

AQ1: AU: A running title was not provided. Please make any necessary changes to this one. AQ2: AU: Please provide the author's name, degree, complete mailing address, and e-mail address. 
AQ3: PE: Need issue/page numbers.

AQ4: Please confirm the authors have no conflicts of interest. 


\section{University Library}

\section{- M M I E E R VA A gateway to Melbourne's research publications}

Minerva Access is the Institutional Repository of The University of Melbourne

Author/s:

Martin, TJ;Seeman, E

Title:

Abaloparatide Is an Anabolic, but Does It Spare Resorption?

Date:

2017-01-01

Citation:

Martin, T. J. \& Seeman, E. (2017). Abaloparatide Is an Anabolic, but Does It Spare

Resorption?. JOURNAL OF BONE AND MINERAL RESEARCH, 32 (1), pp.11-16. https:// doi.org/10.1002/jbmr.3042.

Persistent Link:

http://hdl.handle.net/11343/292223 